\title{
A Prospective Study of Intensity-modified Radiation Therapy in Comparison with Conventional 3D-RT for BR Pancreatic Cancer Patients with Arterial Involvement
}

\author{
TOSHIHIKO MASUI ${ }^{1}$, KYOICHI TAKAORI $^{1}$, TAKAYUKI ANAZAWA ${ }^{1}$, ASAHI SATO ${ }^{1}$, \\ KENZO NAKANO ${ }^{1}$, YUICHIRO UCHIDA ${ }^{1}$, AKITADA YOGO ${ }^{1}$, YOKO GOTO ${ }^{2}$, SHIGEMI MATSUMOTO ${ }^{3}$, \\ YUZO KODAMA $^{4}$, MASASHI KANAI ${ }^{3}$, HIROYOSHI ISODA ${ }^{2}$, MASAKI MIZUMOTO ${ }^{1}$, \\ YOSHIYA KAWAGUCHI ${ }^{1}$, KEIKO SHIBUYA ${ }^{2}$, SATOSHI ITASAKA ${ }^{2}$ and SHINJI UEMOTO ${ }^{1}$ \\ ${ }^{1}$ Department of Surgery, Kyoto University, Kyoto, Japan; \\ ${ }^{2}$ Department of Radiation Oncology and Image-Applied Therapy, Kyoto University, Kyoto, Japan; \\ ${ }^{3}$ Department of Clinical Oncology, Kyoto University, Kyoto, Japan; \\ ${ }^{4}$ Department of Gastroenterology and Hepatology, Kyoto University, Kyoto, Japan
}

\begin{abstract}
Background/Aim: Intensity-modulated radiation therapy (IMRT) is a form of radiation therapy that allows accurate irradiation with reduced damage to surrounding tissues. Here, we analyzed borderline-resectable pancreatic cancer (BRPC) with arterial abutment (BR-A) patients with $I M R T$ as neoadjuvant therapy and performed comparisons with patients with conventional RT to clarify the advantages of IMRT as a neoadjuvant therapy. Patients and Methods: Thirty BR-A patients treated at our hospital between January 2012 and December 2015 were divided into two groups: 12 patients underwent conventional $3 D-R T$ before resection ( $R T$ group); and 18 patients underwent IMRT before resection (IMRT group). We analyzed safety, tumor resection rate, histological classification of the tumor and overall survival. Results: The RO rate was $84 \%$ for the IMRT group and $83 \%$ for the RT group. Local therapeutic effects as assessed by Evans classification showed a higher local control rate in the IMRT group (Grade: 1, 0\%; $2 a$, $25 \% ; 2 b, 41.6 \% ; 3,17 \% ; 4,8 \%)$ than in the $R T$ group (Grade: 1, 17\%; 2a, 50\%; 2b, 17\%; 3, 17\%; 4, 0\%). The cumulative dose of $S 1$ treatment as adjuvant therapy was much smaller in the RT group (18.3\%) compared to that in the IMRT group (57.1\%, p=0.047), and with better subsequent overall survival rate (MST 32 months vs. 13.8 months, $p=0.0273$ ). Conclusion: The IMRT group showed a better control rate than the $R T$ group. The neoadjuvant
\end{abstract}

Correspondence to: Toshihiko Masui, Kyoto University, Department of Surgery, 54 Shogoin, Kawaracho, Sakyo, Kyoto, Japan. Tel: +81 757513242, e-mail: tmasui@kuhp.kyoto-u.ac.jp

Key Words: IMRT, BR-A pancreatic cancer, neoadjuvant.
IMRT has advantages of higher completion rate of adjuvant chemotherapy with better nutritional status and better subsequent overall survival rate (OS).

Pancreatic cancer is one of the most aggressive malignancies, with high fatality rates all over the world (1). This pathology represents the fourth leading cause of death from cancer in Japan, and its incidence is still growing (2). For non-metastatic pancreatic cancer, surgical resection offers the best chance of cure. However, half of patients with pancreatic cancer have distant metastasis on admission and about $30-40 \%$ present with unresectable, locally advanced disease (3). The remaining $20 \%$ of patients can be treated with resection, representing a relatively small fraction of patients (4). To expand the proportion of patients who can undergo resection, the concept of borderline-resectable pancreatic cancer (BRPC) has been proposed (5). The National Comprehensive Cancer Network (NCCN) defines BRPC as a tumor if any of the following occurs: i) focal tumor abutment $\left(<180^{\circ}\right)$ of the superior mesenteric artery (SMA) or of the celiac axis (CA); ii) encasement of common hepatic artery (CHA) but not up to the CA or proper hepatic artery (PHA) borderline resectable pancreatic cancer with arterial abutment (BR-A); or iii) involvement of the superior mesenteric vein (SMV)/portal vein (PV) with abutment larger than $180^{\circ}$ [borderline-resectable pancreatic cancer with venous inclusion (BR-V)]. As a result, BRPC potentially shows difficulty with curative resection, especially for the BR-A category (6), and neoadjuvant therapy with combination therapy with 5-FU, leucovorin, irinotecan and oxaliplatin (FOLFIRINOX) (7), gemcitabine (GEM) plus capecitabine $(8,9)$, or GEM plus radiotherapy (10) are reported to increase survival. However, as the stomach and 
small bowel are located close to the pancreas, both organs are included in the field exposed to radiation (11). Chemoradiotherapy-related gastrointestinal toxicities are major dose-limiting factors, as these toxicities tend to lower quality of life and can even delay treatment of pancreatic cancer (12).

Intensity-modulated radiation therapy (IMRT) is an irradiation therapy that covers customized high-risk volumes while minimizing doses to normal tissue (13). The advantage of this method is reduced toxicity to surrounding normal tissues while maximizing effects in the target volume compared to conventional 3D-RT $(14,15)$. Although IMRT has been used for other sites of disease such as prostate cancer, very little data has been accumulated regarding its use in improving the survival of pancreatic cancer patients $(16,17)$, because of the difficulty in achieving accurate exposure under respiratory movements of the target (18). We have been using IMRT in an effort to achieve more durable local control for patients with medically inoperable, nonmetastatic, locally advanced pancreatic cancer (19).

The present study prospectively compared IMRT and 3DRT treatments for BRPC patients in the same period to investigate pathological effects and toxicity. We also compared intention-to-treat overall survival (OS) between IMRT and 3D-RT patients.

\section{Patients and Methods}

Patients. This study was conducted as an additional study of a phase II study of neoadjuvant treatment with IMRT (UMIN UMIN000010113), which was to evaluate the advantages of IMRT (total dose, 42-45 Gy) as a neoadjuvant therapy compared to the historical surgery first group.

In this study we prospectively compared the survival benefit of IMRT to that of conventional 3D-RT (total dose, $39 \mathrm{~Gy}$ ) in the same period. Resectability was assessed by multidetector-row computed tomography $(\mathrm{CT})$ using a multiphase contrast-enhanced technique. All pancreatic tumors patients who provided consent classified as borderline resectable with arterial attachment (BR-A) according to NCCN guidelines treated at our hospital between January 2012 and December 2015 were enrolled. Patients diagnosed as BR-A pancreatic cancer were allocated to the IMRT group or RT group. The patients who were going to enroll this study initially allocated to IMRT group and if the patients could not be treated with IMRT within 6 weeks from their enrollment because of the limitation of the IMRT branch, they were treated with 3D-RT. About one third of the BR-A patients were allocated to RT group (Figure 1). Inclusion criteria for BR-A pancreatic cancer in terms of resectability in this study were as follows. In brief, patients showing tumor contact with the superior mesenteric artery (SMA) at $\leq 180^{\circ}$ according to the CT image, or tumor contact with the CHA allowing safe and complete resection were defined as BR-A. In addition, tumors in contact with the CA but not extending to the aorta that could be completely resected by distal pancreatectomy with celiac axis resection (DPCAR) were categorized as BR-A. Other inclusion criteria were as follows: histologically or cytologically confirmed pancreatic ductal adenocarcinoma; age $>20$ years; and no distant metastasis found in the thorax, abdomen or pelvis on MRI with contrast medium of gadolinium-ethoxybenzyl diethylenetriaminepentaacetic acid (EOBMRI) or positron emission tomography with 2-deoxy-2-(fluorine18)fluoro- D-glucose (FDG-PET), Zubroad performance status of 0 or 1, no pre-treatment for current pancreatic ductal adenocarcinoma, and no apparent hematological dysfunction or dysfunction of the main organs. Exclusion criteria were as follows: presence of interstitial pneumonitis; history of irradiation to the upper abdomen; history of active cancer within the preceding 3 years; or women of child-bearing age, or expectant or nursing women. The ethics board of Kyoto University approved this study and each patient provided informed consent prior to participation.

Chemoradiotherapy. The protocol for IMRT was as follows. Patients were initially administered gemcitabine 3 times at a dose of $1,000 \mathrm{mg} / \mathrm{m}^{2}$ before chemoradiotherapy. On starting radiotherapy, gemcitabine was administered at a dose of $1,000 \mathrm{mg} / \mathrm{m}^{2}$ (days 1,8 , and 15) concurrent with IMRT. When grade 4 or worse neutrocytopenia or thrombocytopenia occurred, chemotherapy was stopped for a week. We applied 4D-CT simulation for all patients in the current study. During IMRT planning, gross tumor volume (GTV) included the pancreatic tumor and any lymph node $>1 \mathrm{~cm}$ in diameter. Clinical target volume (CTV) was defined as GTV plus a 5 -mm margin as well as the retropancreatic space and the paraaortic lymph nodes between the celiac axis and the superior mesenteric artery. Organs at risk were determined to be the liver, stomach, duodenum, colon and kidney, as well as the spinal cord, and were delineated on expiratory-phase CT. Planning target volume (PTV) was defined as the CTV with a $0.5-\mathrm{cm}$ margin in all directions. IMRT was used to generate optimized treatment plans for each patient. Compensated filters were used in five non-coplanar directions to deliver IMRT treatment. A prescribed dose of 42-45 Gy administered in 15 fractions was determined to be sufficient to cover 95\% of the PTV-boost, a volume that subtracted organ at risk (stomach and duodenum) plus 5-10 mm margin from PTV, whereas keeping PTV minimum dose over $36 \mathrm{~Gy}$.

Surgery and adjuvant chemotherapy. Patients were evaluated for resection at 4 weeks after chemoradiotherapy using MDCT, EOBMRI, and FDG-PET. Resection was attempted between 4 and 12 weeks after completing chemoradiation. In the absence of clear technical unresectability, pancreaticoduodenectomy and distal or total pancreatectomy (and resection of any involved structures) was performed in accordance with the tumor anatomy. In patients scheduled for DP-CAR, preoperative embolization of the CHA was performed to reduce the rate of ischemic complications, as described by Kondo et al. (20). Operative findings, complications, and histopathology were recorded. S-1 at a dose of $80 \mathrm{mg} / \mathrm{m}^{2} /$ day was administered on days 1-28 as adjuvant chemotherapy for 6 months starting 4-8 weeks after resection (21). The cumulative amount was measured according to the patient's prescription record and calculated as ratio to the planned amount. The patients with recurrence less than 6 months after starting adjuvant chemotherapy were excluded for survival analysis.

Assessment. Toxicity events were recorded using the Common Terminology Criteria for Adverse Events (CTCAE version 4.0; http://evs.nci.nih.gov/ftp1/CTCAE/CTCAE_4.03_2010-0614_QuickReference_5x7.pdf). Complete blood count and hepatic function were examined weekly starting from the initiation of radiotherapy until 2 weeks after the completion of chemoradiotherapy. 


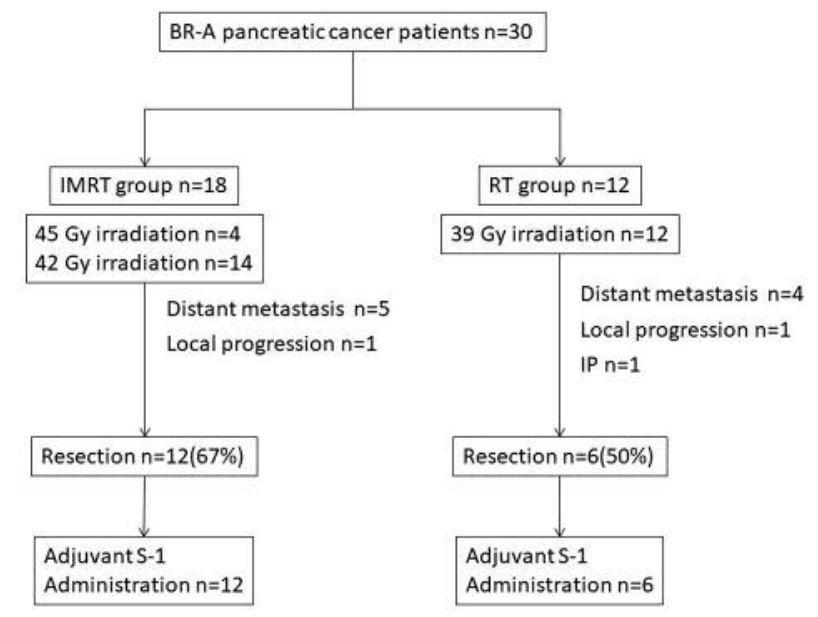

Figure 1. Diagram of NAC study. Surgical resection was performed for $67 \%$ of patients treated with IMRT and 50\% of those with RT.

During the treatment period, patients were examined every 3 weeks. After completion of treatment, follow-up was performed every 4 weeks for the first 6 months, then every 3 months thereafter until death. Serum CEA, CA19-9 and albumin level were observed before and after treatment, and on each follow-up. Serum CA19-9 concentration before treatment was measured after biliary drainage.

Ethical approval. All procedures performed in this study involving human participants were in accordance with the ethical standards of the Kyoto University Institutional Ethical Board and with the 1964 Helsinki declaration and its later amendments or comparable ethical standards. Informed consent was obtained from all individual participants included in the study.

Statistics. OS was calculated from the date of first treatment and estimated using the Kaplan-Meier method. Univariate analysis was conducted with the log-rank test. To identify risk factors independently associated with survivals, multivariate Cox proportional hazards regression analysis was used. The chi-squared test or Fisher's exact test was used to compare categorical variables. Mann-Whitney's $U$ test was applied to compare continuous variables. Values of $p<0.05$ were considered significant. All statistical analyses were performed using JMP version 10.0 software (SAS Institute, Cary, NC).

\section{Results}

Setting of radiation doses and neoadjuvant chemoradiotherapy for BR-A pancreatic cancer. Table I shows the characteristics of patients enrolled in this study between January 2012 and December 2015. We started IMRT at $45 \mathrm{~Gy}$, but found that 2 out of the initial 4 patients showed aneurysm of the artery after resection. We subsequently reduced the irradiation dose to $42 \mathrm{~Gy}$, and no morbidity (Clavien-Dindo 3 or 4) was subsequently encountered. Mean age was 66 years for the IMRT group and 68.5 years for the RT group. Mean tumor size
Table I. Patients characteristics before NAC treatment.

\begin{tabular}{lccc}
\hline & $\begin{array}{c}\text { NAC-IMRT } \\
\mathrm{n}=18\end{array}$ & $\begin{array}{c}\text { NAC-RT } \\
\mathrm{n}=12\end{array}$ & $p$-Value \\
\hline Age(year) & $66(53-77)$ & $68.5(51-79)$ & 0.599 \\
Gender (male:female) & $14: 4$ & $8: 4$ & 0.503 \\
Head/body-tail & $12: 6$ & $9: 3$ & 0.622 \\
Tumor size & $27.75(15.8-47)$ & $28.9(16.6-55.6)$ & 0.401 \\
Arterial involvement & & & \\
CA/CHA & 5 & 3 & 0.866 \\
SMA & 13 & 9 & \\
\hline
\end{tabular}

CA: Celiac axis; CHA: common hepatic artery; SMA: superior mesenteric artery.

was $27.75 \mathrm{~mm}$ in the IMRT group and $28.9 \mathrm{~mm}$ in the RT group. Major sites of arterial involvement were the SMA (IMRT group, $72.2 \%$; RT group, $75 \%$ ). Adverse effect and response evaluations by RECIST criteria at 1 month after NAC treatment are shown in Table II. Grade 3 and 4 adverse events (CTCAE version 4.0 ) were seen in 8 patients $(26.7 \%)$. The most commonly encountered toxicities were hematologic, as either leukocytopenia $(n=6)$ or neutropenia $(n=4)$. Interstitial pneumonitis was seen in 1 patient (3.3\%), who was subsequently removed from the protocol therapy and underwent surgery after pneumonitis treatment. A total of 29 patients $(96.7 \%)$ completed NAC treatment. Response rate was $11 \%$ for IMRT and $16 \%$ for RT, showing no significant difference (Figure 2). However, the reduction rate of serum CA19-9 was significantly higher in the IMRT group (0.45 ratio) compared to that in the NAC-RT group (0.8 ratio, $p=0.048$ ). In contrast, reduction of CEA did not differ significantly between groups. The neutrophil to lymphocyte ratios (NLR) in IMRT group and RT group were 2.85 and 2.37 on average before NAC treatment and 2.63 and 3.56 at one month after NAC treatment, which showed significantly higher NLR in the RT group. On imaging diagnosis, distant metastasis before surgery was seen for 5 patients in the IMRT group (liver metastasis, $\mathrm{n}=3$; peritoneal dissemination, $\mathrm{n}=1$; lung metastasis, $\mathrm{n}=1$ ), and 4 patients in the RT group (liver metastasis, $\mathrm{n}=2$; peritoneal dissemination, $n=2$ ). One patient in each group showed local progression and continued chemotherapy without resection, and a final total of 18 patients underwent resection (pancreaticoduodenectomy, $\mathrm{n}=15$; distal pancreatectomy with celiac axis resection, $n=2$; total pancreatectomy $n=1$ ), resulting in $60 \%$ of the resectability in this study.

Surgical results and pathological effects. As shown in Table III, the rate of the major complication of hemorrhage (Clavien-Dindo IIIa/IIIb) after resection was $16.7 \%$ in IMRT group. However, as noted in the Patients and Methods section, those patients with hemorrhage had been treated 
Table II. Efficacy and adverse events of NAC.

\begin{tabular}{lccc}
\hline & $\begin{array}{c}\text { NAC-IMRT } \\
\mathrm{n}=18\end{array}$ & $\begin{array}{c}\text { NAC-RT } \\
\mathrm{n}=12\end{array}$ & $p$-Value \\
\hline CTCAE grade3 or 4 & 3 & 2 & 0.932 \\
Leucocytopenia & 4 & 2 & \\
Neutoropenia & 2 & 2 & \\
Interstitial pneumonitis & 0 & 1 & \\
Completion rate of chemotherapy & $18(100 \%)$ & $11(91.7 \%)$ & 0.93 \\
Completion rate of radiation & $18(100 \%)$ & $11(91.7 \%)$ & 0.93 \\
RECIST & & & \\
CR & 0 & 0 & 0.259 \\
PR & 2 & 2 & \\
SD & 15 & 8 & \\
PD & 1 & 1 & \\
CEA level decrease ratio & $1.05(0.3-1.7)$ & $0.95(0.3-1.4)$ & 0.985 \\
CA19-9 level decrease ratio & $0.45(0.1-1.5)$ & $0.8(0.2-2.4)$ & 0.048 \\
metastasis after NAC & 5 & 4 & 0.868 \\
liver & 3 & 2 & \\
dissemination & 1 & 2 & \\
lung & 1 & 0 & \\
NLR at enrollment & $2.85 \pm 0.39$ & $2.37 \pm 0.50$ & 0.774 \\
NLR at 1 month after NAC & $2.63 \pm 0.33$ & $3.56 \pm 0.43$ & 0.048 \\
\hline
\end{tabular}

CR: Complete response; PR: partial response; SD: stable disease; PR: progressive disease; NLR: neutrophil to lymphocyte ratio.

Table III. Surgical characteristics.

\begin{tabular}{|c|c|c|c|}
\hline & $\begin{array}{l}\text { NAC-IMRT } \\
\mathrm{n}=12\end{array}$ & $\begin{array}{c}\text { NAC-RT } \\
n=6\end{array}$ & $p$-Value \\
\hline \multicolumn{3}{|l|}{ Operative prodcedure } & 0.406 \\
\hline PD & 9 & 6 & \\
\hline DP & 2 & 0 & \\
\hline $\mathrm{TP}$ & 1 & 0 & \\
\hline SMV/PV resection & $9(75 \%)$ & $5(83.3 \%)$ & 0.108 \\
\hline Major arteiral resection & 2 & 0 & \\
\hline operation time & $476(342-715)$ & $579(453-887)$ & 0.125 \\
\hline Blood loss & $610(225-2780)$ & $910(325-2637)$ & 0.325 \\
\hline \multicolumn{4}{|c|}{ Perioperative complication } \\
\hline Clavien Dindo $>$ IIIa & $2(16.7 \%)$ & $2(33.3 \%)$ & 0.403 \\
\hline Pancreatic fistula (>gradeB) & $4(33.3 \%)$ & $3(50 \%)$ & 0.146 \\
\hline Mortality & $0 \%$ & $0 \%$ & \\
\hline Resectability(R0 rate) & $10(83 \%)$ & $5(83 \%)$ & 1,000 \\
\hline Lymphnode metastasis & $1(8.3 \%)$ & $3(50 \%)$ & 0.045 \\
\hline Evans & & & 0.473 \\
\hline I & $0(0 \%)$ & $1(17 \%)$ & \\
\hline IIa & $4(25 \%)$ & $3(50 \%)$ & \\
\hline $\mathrm{IIb}$ & $5(41.6 \%)$ & $1(17 \%)$ & \\
\hline III & $2(17 \%)$ & $1(17 \%)$ & \\
\hline IV & $1(8 \%)$ & $0(0 \%)$ & \\
\hline $\begin{array}{l}\text { Cumulative dose of GEM per } \\
\text { planned dose before resection }\end{array}$ & $\begin{array}{lc}\mathrm{r} & 91.5 \% \\
\text { on } & (59.4-100)\end{array}$ & $\begin{array}{c}100 \% \\
(67-100)\end{array}$ & 0.385 \\
\hline $\begin{array}{l}\text { Cumulative dose of } \mathrm{S} 1 \text { per } \\
\text { planned dose after resection }\end{array}$ & $\begin{array}{l}56.3 \% \\
(0-100)\end{array}$ & $\begin{array}{c}0 \% \\
(0-60)\end{array}$ & 0.032 \\
\hline $\begin{array}{l}\text { Albumin after } 1 \text { months } \\
(\mathrm{g} / \mathrm{dl})\end{array}$ & $\begin{array}{c}3.15 \\
(2.3-3.9)\end{array}$ & $\begin{array}{c}2.75 \\
(2.1-3.0)\end{array}$ & 0.033 \\
\hline
\end{tabular}

PD: Pancreaticoduodenetomy; DP: distal pancreatectomy; TP: total pancreatectomy.
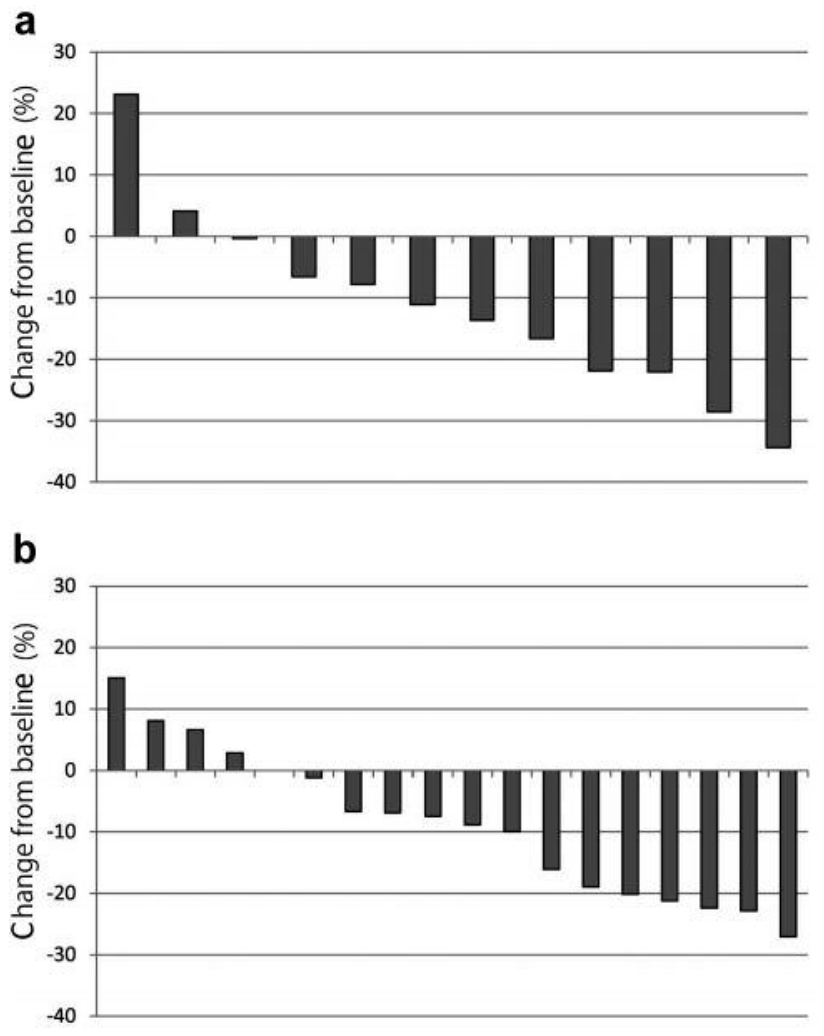

Figure 2. The tumor reduction rate in size during NAC period. A) RT group, B) IMRT group. Total response rate was $11 \%$ for IMRT and $16 \%$ for $R T$.

with IMRT at $45 \mathrm{~Gy}$, and after we reduced the irradiation dose to $42 \mathrm{~Gy}$, no complications over Clavien-Dindo grade IIIa were encountered. Operation time and blood loss were not significantly different between the RT group and IMRT group. No perioperative deaths were seen. The rate of R0 resection was $83 \%$ in both groups. Lymph node metastasis was significantly less in the IMRT group (8.3\%) than in the RT group (50\%, $p=0.045)$. According to the Evans classification as a treatment assessment, the ratio of IIb and above tended to be high in IMRT group. Of note was the finding that the cumulative dose of S-1 per planned dose after resection was significantly higher in the IMRT group than in the RT group $(p=0.047)$. Conversely, cumulative doses of GEM during neoadjuvant treatment did not differ significantly between groups. Serum albumin concentration 1 month after surgery was comparatively higher in the IMRT group than in the RT group ( $p=0.033)$.

Survival. At a median follow-up of 24 months (range $=18-40$ months), median overall survival was 19 months starting from the first treatment (Figure 3). Median overall survival 
a

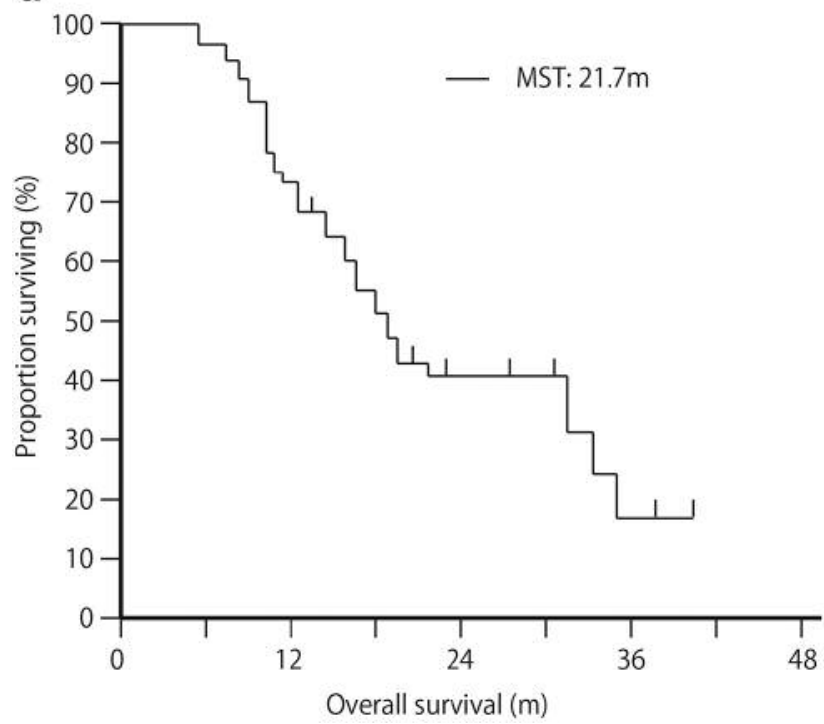

b

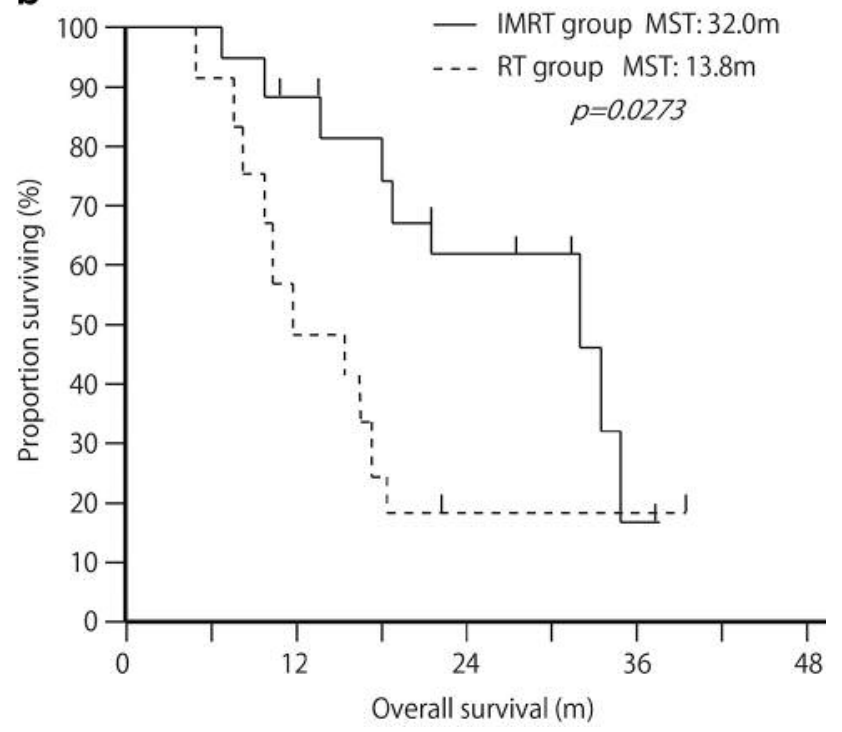

Figure 3. A) Overall survival in 30 patients with BR-A pancreatic cancer. B) Prognosis in patients with IMRT treatment ( $n=18)$ was significantly better than that in patients with RT treatment $(n=12, p=0.0273)$.

Table IV. Factors affecting OS.

\begin{tabular}{|c|c|c|c|c|}
\hline \multirow[b]{2}{*}{ Factors } & \multicolumn{2}{|c|}{ Univariate analysis } & \multicolumn{2}{|c|}{ Multivariate analysis } \\
\hline & Risk ratio $(95 \% \mathrm{CI})$ & $p$-Value & Risk ratio $(95 \% \mathrm{CI})$ & p-Value \\
\hline Gender (male) & $1.23(0.44-4.34)$ & 0.799 & & \\
\hline Age (over 68 years) & $0.83(0.32-2.06)$ & 0.688 & & \\
\hline Tumor over $30 \mathrm{~mm}$ & $0.92(0.32-2.35)$ & 0.876 & & \\
\hline N1 over N0 & $2.19(0.43-10.07)$ & 0.319 & $1.40(0.15-17.91)$ & 0.767 \\
\hline CA19-9 reduction (per ratio) & $0.47(0.81-4.79)$ & 0.12 & & \\
\hline $\mathrm{R} 0$ over $\mathrm{R} 1 / 2$ & $6601(0.63-1.59)$ & 0.111 & & \\
\hline NAC-IMRT over NAC-RT & $0.37(0.14-0.93)$ & 0.036 & $0.903(0.13-12.06)$ & 0.926 \\
\hline Cumulative GEM dose ( $1 \%$ per planned dose) & $0.999(0.96-1.03)$ & 0.971 & & \\
\hline Cumulative $\mathrm{S} 1$ dose ( $1 \%$ per planned dose) & $0.969(0.93-0.99)$ & 0.021 & $0.972(0.933-0.998)$ & 0.039 \\
\hline
\end{tabular}

of patients with IMRT was 32 months, compared to 13.8 months with RT, suggesting significantly longer survival in the IMRT group ( $p=0.0273, \log$-rank test). When we analyzed factors affecting overall survival of BR-A PDAC patients (Table IV), univariate analysis showed IMRT and cumulative S-1 dose as adjuvant therapy as factors affecting overall survival ( $p=0.036$ and $p=0.021$, respectively). In multivariate analysis, cumulative dose per planned amount of S-1 administration was an independent risk factor for better overall survival $(p=0.039)$. To date, 11 of 18 resected patients experienced recurrence, and 5 patients (44\%) had liver metastasis, 3 patients $(28 \%)$ had peritoneal dissemination, 2 patients (18\%) had lung metastasis and 1 patient $(9 \%)$ who had undergone $\mathrm{R} 1$ resection experienced local recurrence at the primary tumor site.

\section{Discussion}

This study investigated the advantages of IMRT over RT for BR-A pancreatic cancer as a neoadjuvant therapy. Although tumor reduction on imaging did not show any significant difference between groups, serum CA19-9 levels were significantly lower in the IMRT group. Pathological effects were broadly similar in both groups, but cumulative S-1 dose 
per planned dose was significantly higher in the IMRT group $(57.1 \%)$ than in the RT group $(18.3 \%, p=0.047)$. Interestingly, a risk factor for short OS in multivariate analysis was lower cumulative $\mathrm{S}-1$ dose.

NCCN guidelines suggest consideration of neoadjuvant treatment for patients with BRPC (5), because of the high risk of margin-positive resection. Indeed, survival data for patient's resection of BRPC, specifically BRPC with arterial involvement, from the Japan Pancreatic Society showed a 5year OS rate of only 5\% (3). Many trials have been conducted with the intention of improving such outcomes, and neoadjuvant treatment including ours has been demonstrated to improve the $\mathrm{R} 0$ resection rate for patients with $\operatorname{BRPC}(10,22-24)$. However, although $\mathrm{R} 0$ resection is important for improving survival after resection, distant metastasis is the most common form of recurrence after resection $(10,24)$. In the present series, no significant relationship was observed between R0 rate and OS. From this perspective, control of both local tumors and distant metastasis need to be considered in the treatment of patients with BRPC. We have therefore introduced IMRT with full-dose GEM treatment for neoadjuvant therapy to patients showing arterial involvement of BRPC.

IMRT may provide several advantages for the treatment of pancreatic cancer in neoadjuvant settings. Several dosimetric studies have shown reduced doses to the kidneys, stomach, liver and small bowel with IMRT as compared to traditional $3 \mathrm{D}$-confocal field radiation therapy $(25,26)$. In the postoperative adjuvant setting, IMRT appears associated with minimal treatment breaks and reductions in rates of Grade 3 or 4 nausea and diarrhea compared to 3D-confocal treatment (25). In our series, neoadjuvant therapy was well tolerated with the use of IMRT (completion rate=96.7\%). We applied a strategy for IMRT to reduce the treatment period by increasing the radiation exposure per dose, and the effect of such radiation therapy has been reported as 20-30\% higher per Gray compared to the usual fractionated irradiation (19). In principle, reductions in the dose to and exposed volume of normal tissue may increase the tolerance of concurrent chemoradiation and allow more intensive regimens. We previously administered a 3D-RT dose of 42 Gy but failed to complete treatment because of comorbidities, necessitating subsequent reduction to $39 \mathrm{~Gy}$. A dose of 39 Gy may not be sufficient for R0 resection even with full-dose gemcitabine. As NAC treatment, IMRT with the dose increased to $42 \mathrm{~Gy}$ is more appropriate.

In the current NAC series, survival was significantly better in IMRT patients than in RT patients. Median OS was 32 months in IMRT patients, compared to 13.8 months in RT patients. Neither R0 rate nor Evans grade differed significantly between groups, although the effects tended to be slightly better in the IMRT group. Cumulative dose of GEM and rate of adverse events were similar between groups. However, a significant difference was seen in cumulative adjuvant chemotherapy dose. Cumulative adjuvant chemotherapy dose has been reported as significantly related to survival in patients with potentially resectable pancreatic cancer under a surgery-first approach (27). Moreover, most cases of recurrence in this study involved distant metastasis, suggesting the necessity of sufficient adjuvant chemotherapy. In addition, when we compared nutritional status as represented by serum albumin, the IMRT group appeared better nourished. Okumura et al. (28) recently published a report showing that preoperative nutritional status relates with survival after resection and Matsumoto et al. (29) reported that the post-operative albumin is a marker of incomplete adjuvant chemotherapy. Moreover, our analysis about NLR after NAC showed significantly higher NLR in RT group compared to IMRT group. Recently, Yang et al. (30) showed high peripheral blood NLR suggested a poor prognosis for patient with pancreatic cancer in meta-analysis. The systemic inflammatory response from cancer cells promotes the infiltration of neutrophils, which provides a favorable tumor environment for cancer progression and in contrast, lymphocytes are important in the immune defense against cancer cells (31). Although other factors are obviously involved, better nutritional as well as immunological status after IMRT in conjunction with cumulative dose of adjuvant chemotherapy may be part of the reason for better survival after neoadjuvant IMRT.

In this study, 40-50\% of BRPC patients could not undergo resection after NAC treatment because of distant metastasis. Our protocol did not include the use of preoperative laparotomy, which might have contributed to the high rate of distant metastasis after neoadjuvant treatment. In contrast, we encountered only $5-10 \%$ of local progression necessitating in abandonment of resection. In this sense, more rigorous chemotherapeutic therapy before resection might be appropriate as NAC treatment. NAC treatments comprising of gemcitabine/nab-paclitaxel/S-1 combination (23) or FOLFIRINOX (7) therapy have recently been reported to show tolerable adverse events, although intensified chemotherapy may lead to adverse events in combination with irradiation. Nagakawa et al. reported results of NACIMRT with GEM+S1 (32) and a protocol of 50.4-Gy IMRT with GEM at $600 \mathrm{mg} / \mathrm{m}^{2}$ and S-1 at $60 \mathrm{mg}$, lower than our current GEM dose of $1,000 \mathrm{mg} / \mathrm{m}^{2}$, but with addition of $\mathrm{S}-1$. They found that all recurrences were at distant sites, as in the present study. As such, the balance between irradiation and strength of chemotherapy should be considered in the future, and one strategy may be to increase the strength of induction chemotherapy. Even so, application of IMRT instead of 3DRT appears reasonable to reduce adverse events while maintaining control of local progression.

To the best of our knowledge, this represents the first analysis comparing IMRT and 3D-RT in the neoadjuvant setting for BRA pancreatic cancer. However, several limitations must be considered when interpreting the present results. First, although this study was prospective in design and no differences were 
seen in the background characteristics of patients, allocations to NAC treatment were not fully randomized. All BR-A patients not meeting exclusion criteria were eligible for neoadjuvant chemoradiotherapy, with the allocation of radiation methods only dependent on the capacity of the IMRT branch. Second, the sample size was comparatively small to provide thorough statistical assessment of chemoradiation effects. As the benefits of IMRT have gradually been reinforced, we have been applying IMRT for all BRPC patients since 2016, and no patients have been treated with 3D-RT since this date. The present study, therefore, compared and evaluated efficacy in patients who had undergone NAC treatment during the period from 2012 to 2015.

In conclusion, both neoadjuvant treatment with IMRT and 3D-RT offer favorable efficacy and margin-negative status for patients with BR-A pancreatic cancer. However, patients treated using IMRT received higher cumulative doses of S-1 and showed better nutritional status, which might have contributed to significantly longer survival among patients treated using IMRT. Most recurrences were seen at distant sites, suggesting the importance of the balance between intensities of chemo- and radiotherapy. IMRT offers the advantages of relatively low toxicity and favorable conditions for combination with more intense chemotherapy.

\section{Conflicts of Interest}

All Authors declare that they have no conflicts of interest regarding this study.

\section{References}

1 Siegel RL, Miller KD and Jemal A: Cancer statistics, 2016. CA Cancer J Clin 66(1): 7-30, 2016.

2 Ministry of Health Law and Welfare: The dynamic statistics of the population in 2015. http://wwwmhlwgojp/toukei/saikin/hw/ jinkou/kakutei15/index.html, 2016.

3 Matsuno S, Egawa S, Fukuyama S, Motoi F, Sunamura M, Isaji S, Imaizumi T, Okada S, Kato H, Suda K, Nakao A, Hiraoka T, Hosotani R and Takeda K: Pancreatic cancer registry in japan: 20 years of experience. Pancreas 28(3): 219-230, 2004.

4 Stathis A and Moore MJ: Advanced pancreatic carcinoma: Current treatment and future challenges. Nat Rev Clin Oncol 7(3): 163-172, 2010.

5 National Comprehensive Cancer Network: NCCN clinical practice guidelines in oncology (NCCN guidelines). https:// wwwneenorg/professionalsphysician_gls/pdf/pancreatic.pdf, 2016.

6 Bockhorn M, Uzunoglu FG, Adham M, Imrie C, Milicevic M, Sandberg AA, Asbun HJ, Bassi C, Buchler M, Charnley RM, Conlon K, Cruz LF, Dervenis C, Fingerhutt A, Friess H, Gouma DJ, Hartwig W, Lillemoe KD, Montorsi M, Neoptolemos JP, Shrikhande SV, Takaori K, Traverso W, Vashist YK, Vollmer C, Yeo CJ, Izbicki JR and International Study Group of Pancreatic S: Borderline resectable pancreatic cancer: A consensus statement by the international study group of pancreatic surgery (isgps). Surgery 155(6): 977-988, 2014.
7 Suker M, Beumer BR, Sadot E, Marthey L, Faris JE, Mellon EA, El-Rayes BF, Wang-Gillam A, Lacy J, Hosein PJ, Moorcraft SY, Conroy T, Hohla F, Allen P, Taieb J, Hong TS, Shridhar R, Chau I, van Eijck CH and Groot Koerkamp B: Folfirinox for locally advanced pancreatic cancer: A systematic review and patient-level meta-analysis. Lancet Oncol 17(6): 801-810, 2016.

8 Herrmann R, Bodoky G, Ruhstaller T, Glimelius B, Bajetta E, Schuller J, Saletti P, Bauer J, Figer A, Pestalozzi B, Kohne CH, Mingrone W, Stemmer SM, Tamas K, Kornek GV, Koeberle D, Cina S, Bernhard J, Dietrich D, Scheithauer W, Swiss Group for Clinical Cancer R and Central European Cooperative Oncology G: Gemcitabine plus capecitabine compared with gemcitabine alone in advanced pancreatic cancer: A randomized, multicenter, phase iii trial of the swiss group for clinical cancer research and the central european cooperative oncology group. J Clin Oncol 25(16): 2212-2217, 2007.

9 Cunningham D, Chau I, Stocken DD, Valle JW, Smith D, Steward W, Harper PG, Dunn J, Tudur-Smith C, West J, Falk S, Crellin A, Adab F, Thompson J, Leonard P, Ostrowski J, Eatock M, Scheithauer W, Herrmann R and Neoptolemos JP: Phase iii randomized comparison of gemcitabine versus gemcitabine plus capecitabine in patients with advanced pancreatic cancer. J Clin Oncol 27(33): 5513-5518, 2009.

10 Takahashi H, Akita H, Tomokuni A, Kobayashi S, Ohigashi H, Fijiwara Y, Yano M, Sakon M and Ishikawa O: Preoperative gemcitabine-based chemoradiation therapy for borderline resectable pancreatic cancer: Impact of venous and arterial involvement status on surgical outcome and pattern of recurrence. Ann Surg 264(6): 1091-1097, 2016.

11 Kavanagh BD, Pan CC, Dawson LA, Das SK, Li XA, Ten Haken RK and Miften M: Radiation dose-volume effects in the stomach and small bowel. Int J Radiat Oncol Biol Phys 76(3 Suppl): S101-107, 2010.

12 Nakamura A, Shibuya K, Matsuo Y, Nakamura M, Shiinoki T, Mizowaki T and Hiraoka M: Analysis of dosimetric parameters associated with acute gastrointestinal toxicity and upper gastrointestinal bleeding in locally advanced pancreatic cancer patients treated with gemcitabine-based concurrent chemoradiotherapy. Int J Radiat Oncol Biol Phys 84(2): 369-375, 2012.

13 Huang X, Knoble JL, Zeng M, Aguila FN, Patel T, Chambers $\mathrm{LW}, \mathrm{Hu} \mathrm{H}$ and Liu $\mathrm{H}$ : Neoadjuvant gemcitabine chemotherapy followed by concurrent imrt simultaneous boost achieves high r0 resection in borderline resectable pancreatic cancer patients. PLoS One 11(12): e0166606, 2016.

14 Lee KJ, Yoon HI, Chung MJ, Park JY, Bang S, Park SW, Seong JS and Song SY: A comparison of gastrointestinal toxicities between intensity-modulated radiotherapy and three-dimensional conformal radiotherapy for pancreatic cancer. Gut Liver 10(2): 303-309, 2016.

15 Taylor R, Opfermann K, Jones BD, Terwilliger LE, McDonald DG, Ashenafi MS, Garrett-Meyer E and Marshall DT: Comparison of radiation treatment delivery for pancreatic cancer: Linac intensity-modulated radiotherapy versus helical tomotherapy. J Med Imaging Radiat Oncol 56(3): 332-337, 2012.

16 Combs SE, Habermehl D, Kessel K, Bergmann F, Werner J, Brecht I, Schirmacher P, Jager D, Buchler MW and Debus J: Intensity modulated radiotherapy as neoadjuvant chemoradiation for the treatment of patients with locally advanced pancreatic cancer. Outcome analysis and comparison with a 3d-treated patient cohort. Strahlenther Onkol 189(9): 738-744, 2013. 
17 Petelska AD, Naumowicz $M$ and Figaszewski ZA: The equilibrium of phosphatidylcholine-amino acid system in monolayer at the air/water interface. Cell Biochem Biophys 60(3): 155-160, 2011.

18 Mori S, Hara R, Yanagi T, Sharp GC, Kumagai M, Asakura H, Kishimoto R, Yamada S, Kandatsu S and Kamada T: Fourdimensional measurement of intrafractional respiratory motion of pancreatic tumors using a 256 multi-slice ct scanner. Radiother Oncol 92(2): 231-237, 2009.

19 Nakamura A, Shibuya K, Nakamura M, Matsuo Y, Shiinoki T, Nakata M, Mizowaki T and Hiraoka M: Interfractional dose variations in the stomach and the bowels during breathhold intensity-modulated radiotherapy for pancreatic cancer: Implications for a dose-escalation strategy. Med Phys 40(2): 021701, 2013.

20 Kondo S, Katoh H, Shimizu T, Omi M, Hirano S, Ambo Y, Okushiba $S$ and Morikawa T: Preoperative embolization of the common hepatic artery in preparation for radical pancreatectomy for pancreas body cancer. Hepatogastroenterology 47(35): 14471449,2000

21 Uesaka K, Boku N, Fukutomi A, Okamura Y, Konishi M, Matsumoto I, Kaneoka Y, Shimizu Y, Nakamori S, Sakamoto H, Morinaga S, Kainuma O, Imai K, Sata N, Hishinuma S, Ojima H, Yamaguchi R, Hirano S, Sudo T, Ohashi Y and Group JS: Adjuvant chemotherapy of s-1 versus gemcitabine for resected pancreatic cancer: A phase 3, open-label, randomised, noninferiority trial (jaspac 01). Lancet 388(10041): 248-257, 2016.

22 Masui T, Doi R, Kawaguchi Y, Sato A, Nakano K, Ito T, Anazawa $\mathrm{T}$, Takaori $\mathrm{K}$ and Uemoto $\mathrm{S}$ : Concurrent gemcitabine+s-1 neoadjuvant chemotherapy contributes to the improved survival of patients with small borderline-resectable pancreatic cancer tumors. Surg Today 46(11): 1282-1289, 2016.

23 Kondo N, Murakami Y, Uemura K, Sudo T, Hashimoto Y, Nakagawa $\mathrm{N}$, Takahashi $\mathrm{S}$, Ohge $\mathrm{H}$ and Sueda $\mathrm{T}$ : A phase 1 study of gemcitabine/nab-paclitaxel/s-1 (gas) combination neoadjuvant chemotherapy for patients with locally advanced pancreatic adenocarcinoma. Cancer Chemother Pharmacol 79(4): 775-781, 2017.

24 Mayo SC, Nathan H, Cameron JL, Olino K, Edil BH, Herman JM, Hirose K, Schulick RD, Choti MA, Wolfgang CL and Pawlik TM: Conditional survival in patients with pancreatic ductal adenocarcinoma resected with curative intent. Cancer 118(10): 2674-2681, 2012.

25 Yovino S, Poppe M, Jabbour S, David V, Garofalo M, Pandya $\mathrm{N}$, Alexander R, Hanna N and Regine WF: Intensity-modulated radiation therapy significantly improves acute gastrointestinal toxicity in pancreatic and ampullary cancers. Int $\mathrm{J}$ Radiat Oncol Biol Phys 79(1): 158-162, 2011.
26 Petit SF, Wu B, Kazhdan M, Dekker A, Simari P, Kumar R, Taylor R, Herman JM and McNutt T: Increased organ sparing using shape-based treatment plan optimization for intensity modulated radiation therapy of pancreatic adenocarcinoma. Radiother Oncol 102(1): 38-44, 2012.

27 Yabusaki N, Fujii T, Yamada S, Murotani K, Sugimoto H, Kanda M, Nakayama G, Koike M, Fujiwara M and Kodera Y: The significance of relative dose intensity in adjuvant chemotherapy of pancreatic ductal adenocarcinoma-including the analysis of clinicopathological factors influencing relative dose intensity. Medicine (Baltimore) 95(29): e4282, 2016.

28 Okumura S, Kaido T, Hamaguchi Y, Fujimoto Y, Masui T, Mizumoto M, Hammad A, Mori A, Takaori K and Uemoto S: Impact of preoperative quality as well as quantity of skeletal muscle on survival after resection of pancreatic cancer. Surgery 157(6): 1088-1098, 2015

29 Matsumoto I, Tanaka M, Shirakawa S, Shinzeki M, Toyama H, Asari S, Goto T, Yamashita H, Ishida J, Ajiki T, Fukumoto T, Shimokawa $\mathrm{M}$ and $\mathrm{Ku} \mathrm{Y}$ : Postoperative serum albumin level is a marker of incomplete adjuvant chemotherapy in patients with pancreatic ductal adenocarcinoma. Ann Surg Oncol 22(7): 24082415, 2015.

30 Yang JJ, Hu ZG, Shi WX, Deng T, He SQ and Yuan SG: Prognostic significance of neutrophil to lymphocyte ratio in pancreatic cancer: A meta-analysis. World J Gastroenterol 21(9): 2807-2815, 2015.

31 Dunn GP, Old LJ and Schreiber RD: The immunobiology of cancer immunosurveillance and immunoediting. Immunity 21(2): 137-148, 2004.

32 Nagakawa Y, Hosokawa Y, Nakayama H, Sahara Y, Takishita C, Nakajima T, Hijikata Y, Kasuya K, Katsumata K, Tokuuye K and Tsuchida A: A phase ii trial of neoadjuvant chemoradiotherapy with intensity-modulated radiotherapy combined with gemcitabine and s- 1 for borderline-resectable pancreatic cancer with arterial involvement. Cancer Chemother Pharmacol 79(5): 951-957, 2017. 